Supporting Information

\title{
Comparative Evaluation of the Liquid-Liquid Equilibria of the Extraction of Valeric or Caproic acids from Water by Esters
}

\author{
Ali Ghanadzadeh Gilani*, Elham Nasiri-Touli \\ Department of Chemistry, Faculty of Science, University of Guilan, Rasht 41335-1914, Iran
}

* Corresponding author

E-mail address: aggilani@guilan.ac.ir (A. Ghanadzadeh Gilani)

Tel/Fax: +981313233262

Address Department of Chemistry, Faculty of Science, University of Guilan, Rasht 41335-1914, Iran 
Table S1 Equations for Refractive Index of the Equilibrium Compositions $\left(w_{1}, w_{2}\right.$, and $\left.w_{3}\right)$ Correspond to the Solubility Data in Organic Phase $\left(n_{D}\right)$ for the [Water $(1)+$ Valeric or Caproic Acids (2) + Ester (3)] Systems at $T=298.2 \mathrm{~K}$ and $p=101.3 \mathrm{kPa} .{ }^{\text {a }}$

\begin{tabular}{|c|c|c|c|c|c|}
\hline \multicolumn{4}{|c|}{ Organic phase } & \multirow[t]{2}{*}{ Equation } & \multirow[t]{2}{*}{$\mathrm{R}^{2}$} \\
\hline$w_{1}$ & $w_{2}$ & $w_{3}$ & $n_{D}$ & & \\
\hline \multicolumn{4}{|c|}{ Water (1) + Valeric Acid (2) + Cyclohexyl Acetate (3) } & $\mathrm{n}_{\mathrm{D}}=0.0342 w_{3}+1.4019$ & 0.9991 \\
\hline 0.016 & 0.053 & 0.931 & 1.4340 & & \\
\hline 0.022 & 0.096 & 0.882 & 1.4320 & & \\
\hline 0.027 & 0.146 & 0.827 & 1.4300 & & \\
\hline 0.044 & 0.289 & 0.667 & 1.4250 & & \\
\hline 0.053 & 0.376 & 0.571 & 1.4215 & & \\
\hline 0.063 & 0.462 & 0.475 & 1.4181 & & \\
\hline \multicolumn{4}{|c|}{ Water (1) + Valeric Acid (2) + Benzyl Acetate (3) } & $\mathrm{n}_{\mathrm{D}}=0.0930 w_{3}+1.4050$ & 0.9999 \\
\hline 0.017 & 0.050 & 0.933 & 1.4920 & & \\
\hline 0.025 & 0.099 & 0.876 & 1.4864 & & \\
\hline 0.037 & 0.192 & 0.771 & 1.4768 & & \\
\hline 0.048 & 0.284 & 0.668 & 1.4669 & & \\
\hline 0.061 & 0.373 & 0.566 & 1.4577 & & \\
\hline 0.073 & 0.458 & 0.469 & 1.4488 & & \\
\hline \multicolumn{4}{|c|}{ Water (1) + Valeric Acid (2) + Benzyl Benzoate (3) } & $\mathrm{n}_{\mathrm{D}}=0.1550 w_{3}+1.4045$ & 0.9997 \\
\hline 0.007 & 0.052 & 0.941 & 1.5505 & & \\
\hline 0.009 & 0.097 & 0.894 & 1.5431 & & \\
\hline 0.017 & 0.195 & 0.788 & 1.5271 & & \\
\hline 0.027 & 0.287 & 0.686 & 1.5100 & & \\
\hline 0.035 & 0.381 & 0.584 & 1.4950 & & \\
\hline 0.045 & 0.464 & 0.491 & 1.4810 & & \\
\hline \multicolumn{4}{|c|}{ Water (1) + Caproic Acid (2) + Cyclohexyl Acetate (3) } & $\mathrm{n}_{\mathrm{D}}=0.0264 w_{3}+1.4106$ & 0.9994 \\
\hline 0.013 & 0.053 & 0.934 & 1.4352 & & \\
\hline 0.018 & 0.097 & 0.885 & 1.4340 & & \\
\hline 0.028 & 0.192 & 0.78 & 1.4310 & & \\
\hline 0.036 & 0.287 & 0.677 & 1.4285 & & \\
\hline 0.044 & 0.377 & 0.579 & 1.4260 & & \\
\hline 0.051 & 0.474 & 0.475 & 1.4230 & & \\
\hline \multicolumn{4}{|c|}{ Water (1) + Caproic Acid (2) + Benzyl Acetate (3) } & $\mathrm{n}_{\mathrm{D}}=0.0907 w_{3}+1.4088$ & 0.9979 \\
\hline 0.016 & 0.054 & 0.930 & 1.4941 & & \\
\hline 0.022 & 0.103 & 0.875 & 1.4880 & & \\
\hline 0.033 & 0.196 & 0.771 & 1.4779 & & \\
\hline 0.044 & 0.288 & 0.668 & 1.4687 & & \\
\hline 0.051 & 0.372 & 0.577 & 1.4610 & & \\
\hline 0.058 & 0.474 & 0.468 & 1.4520 & & \\
\hline \multicolumn{4}{|c|}{ Water (1) + Caproic Acid (2) + Benzyl Benzoate (3) } & $\mathrm{n}_{\mathrm{D}}=0.1652 w_{3}+1.4003$ & 0.9994 \\
\hline 0.003 & 0.049 & 0.948 & 1.5570 & & \\
\hline 0.005 & 0.098 & 0.897 & 1.5492 & & \\
\hline 0.009 & 0.195 & 0.796 & 1.5311 & & \\
\hline 0.016 & 0.294 & 0.690 & 1.5141 & & \\
\hline 0.024 & 0.385 & 0.591 & 1.4970 & & \\
\hline 0.031 & 0.480 & 0.489 & 1.4820 & & \\
\hline
\end{tabular}

a Standard uncertainties $\mathrm{u}$ are $\mathrm{u}(T)=0.1 \mathrm{~K}, \mathrm{u}\left(n_{D}\right)=0.0005, \mathrm{u}(p)=1 \mathrm{kPa}$, and $\mathrm{u}(w)=0.006$. 
Table S2 Hand (a and b) Constants and the Correlation Factor $\left(R^{2}\right)$ for (Water - Valeric and Caproic Acids - Ester) Systems at $T=298.2 \mathrm{~K}$ and $p=101.3 \mathrm{kPa}$.

\begin{tabular}{|c|c|c|c|}
\hline \multirow[t]{2}{*}{ Ternary system } & \multicolumn{3}{|c|}{ Hand correlation } \\
\hline & $\mathrm{a}$ & $\mathrm{b}$ & $\mathrm{R}^{2}$ \\
\hline [water (1) + valeric acid (2) + cyclohexyl acetate (3)] & -3.8920 & 0.4502 & 0.9932 \\
\hline$[$ water $(1)+$ valeric acid $(2)+$ benzyl acetate $(3)]$ & -3.6561 & 0.3173 & 0.9973 \\
\hline [water (1) + valeric acid (2) benzyl benzoate (3)] & -3.5813 & 0.3535 & 0.9948 \\
\hline [water $(1)+$ caproic acid (2) + cyclohexyl acetate (3)] & -5.0630 & 0.4765 & 0.9993 \\
\hline [water $(1)+$ caproic acid (2) + benzyl acetate (3)] & -4.5201 & 0.3268 & 0.9993 \\
\hline [water $(1)+$ caproic acid (2) + benzyl benzoate (3)] & -4.7013 & 0.3101 & 0.9984 \\
\hline
\end{tabular}


Table S3 Calculated UNIQUAC and NRTL Tie-Line Data for the (Water - Valeric or Caproic Acids - Esters) Ternary Systems at $T=298.2 \mathrm{~K}$.

\begin{tabular}{|c|c|c|c|c|c|c|c|}
\hline \multicolumn{4}{|c|}{ Aqueous phase (mass fraction) } & \multicolumn{4}{|c|}{ Organic phase (mass fraction) } \\
\hline \multicolumn{2}{|c|}{$\mathrm{w}_{11}$} & \multicolumn{2}{|c|}{$\mathrm{w}_{21}$} & \multicolumn{2}{|c|}{$\mathrm{w}_{13}$} & \multicolumn{2}{|c|}{$\mathrm{W}_{23}$} \\
\hline UNIQUAC & NRTL & UNIQUAC & NRTL & UNIQUAC & NRTL & UNIQUAC & NRTL \\
\hline \multicolumn{8}{|c|}{ water + valeric acid + cyclohexyl acetate } \\
\hline 0.985 & 0.991 & 0.010 & 0.006 & 0.039 & 0.034 & 0.192 & 0.196 \\
\hline 0.980 & 0.984 & 0.015 & 0.013 & 0.051 & 0.049 & 0.325 & 0.327 \\
\hline 0.978 & 0.979 & 0.018 & 0.019 & 0.059 & 0.059 & 0.405 & 0.404 \\
\hline 0.977 & 0.973 & 0.019 & 0.025 & 0.067 & 0.068 & 0.475 & 0.472 \\
\hline 0.977 & 0.969 & 0.020 & 0.029 & 0.072 & 0.075 & 0.525 & 0.521 \\
\hline 0.976 & 0.966 & 0.020 & 0.032 & 0.075 & 0.080 & 0.560 & 0.555 \\
\hline \multicolumn{8}{|c|}{ water + valeric acid + benzyl acetate } \\
\hline 0.975 & 0.980 & 0.020 & 0.015 & 0.038 & 0.042 & 0.213 & 0.216 \\
\hline 0.971 & 0.974 & 0.025 & 0.022 & 0.050 & 0.054 & 0.309 & 0.309 \\
\hline 0.969 & 0.968 & 0.026 & 0.029 & 0.066 & 0.068 & 0.420 & 0.418 \\
\hline 0.969 & 0.965 & 0.026 & 0.032 & 0.074 & 0.075 & 0.470 & 0.468 \\
\hline 0.969 & 0.961 & 0.026 & 0.036 & 0.084 & 0.083 & 0.533 & 0.531 \\
\hline 0.970 & 0.958 & 0.026 & 0.040 & 0.091 & 0.091 & 0.588 & 0.586 \\
\hline \multicolumn{8}{|c|}{ water + valeric acid + benzyl benzoate } \\
\hline 0.974 & 0.992 & 0.024 & 0.007 & 0.018 & 0.028 & 0.181 & 0.193 \\
\hline 0.972 & 0.982 & 0.026 & 0.017 & 0.035 & 0.041 & 0.325 & 0.327 \\
\hline 0.978 & 0.973 & 0.019 & 0.026 & 0.042 & 0.049 & 0.416 & 0.409 \\
\hline 0.978 & 0.966 & 0.019 & 0.033 & 0.048 & 0.055 & 0.480 & 0.472 \\
\hline 0.977 & 0.959 & 0.020 & 0.041 & 0.053 & 0.062 & 0.548 & 0.537 \\
\hline 0.977 & 0.954 & 0.020 & 0.045 & 0.057 & 0.069 & 0.597 & 0.585 \\
\hline \multicolumn{8}{|c|}{ water + caproic acid + cyclohexyl acetate } \\
\hline 0.991 & 0.992 & 0.004 & 0.005 & 0.034 & 0.023 & 0.207 & 0.209 \\
\hline 0.990 & 0.991 & 0.006 & 0.005 & 0.041 & 0.033 & 0.340 & 0.342 \\
\hline 0.989 & 0.991 & 0.007 & 0.006 & 0.047 & 0.042 & 0.435 & 0.438 \\
\hline 0.989 & 0.991 & 0.007 & 0.006 & 0.050 & 0.046 & 0.487 & 0.489 \\
\hline 0.989 & 0.991 & 0.007 & 0.006 & 0.052 & 0.051 & 0.544 & 0.545 \\
\hline 0.989 & 0.991 & 0.007 & 0.007 & 0.054 & 0.055 & 0.593 & 0.592 \\
\hline \multicolumn{8}{|c|}{ water + caproic acid + benzyl acetate } \\
\hline 0.986 & 0.989 & 0.009 & 0.006 & 0.032 & 0.028 & 0.206 & 0.209 \\
\hline 0.984 & 0.988 & 0.012 & 0.007 & 0.042 & 0.037 & 0.327 & 0.331 \\
\hline 0.984 & 0.988 & 0.012 & 0.007 & 0.052 & 0.044 & 0.424 & 0.429 \\
\hline 0.984 & 0.988 & 0.011 & 0.007 & 0.060 & 0.050 & 0.492 & 0.498 \\
\hline 0.984 & 0.988 & 0.011 & 0.008 & 0.065 & 0.054 & 0.542 & 0.549 \\
\hline 0.984 & 0.988 & 0.011 & 0.008 & 0.068 & 0.056 & 0.567 & 0.574 \\
\hline \multicolumn{8}{|c|}{ water + caproic acid + benzyl benzoate } \\
\hline 0.989 & 0.992 & 0.008 & 0.005 & 0.017 & 0.011 & 0.208 & 0.211 \\
\hline 0.989 & 0.992 & 0.007 & 0.006 & 0.026 & 0.021 & 0.323 & 0.326 \\
\hline 0.989 & 0.991 & 0.007 & 0.006 & 0.034 & 0.031 & 0.432 & 0.433 \\
\hline 0.989 & 0.991 & 0.007 & 0.007 & 0.036 & 0.037 & 0.489 & 0.488 \\
\hline 0.989 & 0.991 & 0.007 & 0.007 & 0.038 & 0.041 & 0.533 & 0.531 \\
\hline 0.989 & 0.991 & 0.006 & 0.007 & 0.040 & 0.046 & 0.576 & 0.572 \\
\hline
\end{tabular}




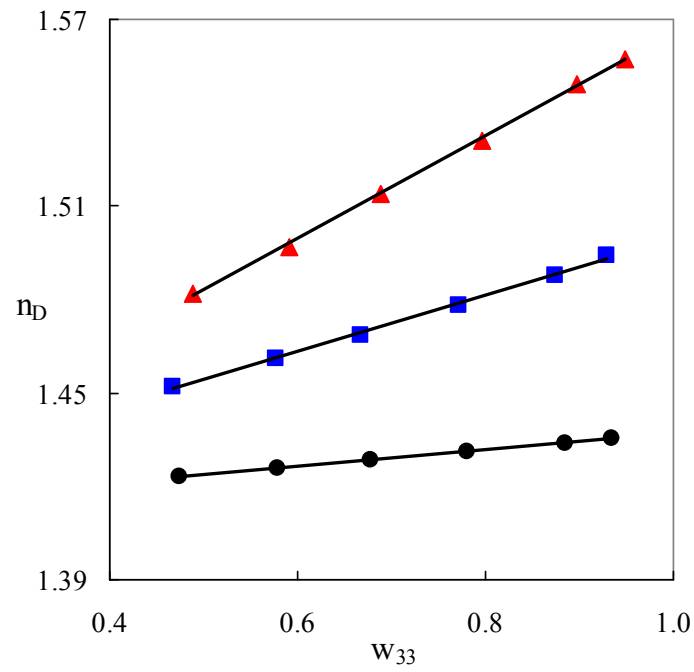

(a)

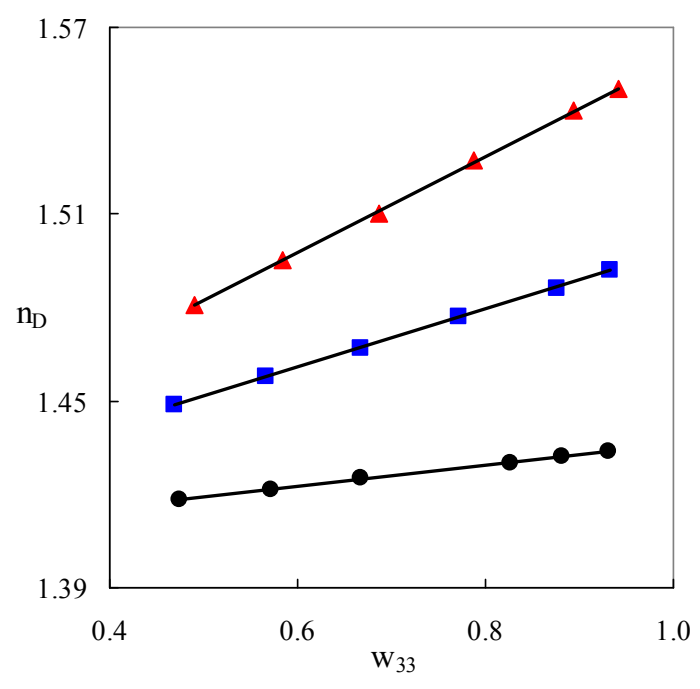

(b)

Figure S1 Refractive index standard curves for \{water - (a) valeric acid and (b) caproic acid cyclic esters\} ternary systems; $(\circ)$ cyclohexyl acetate, $(\Delta)$ benzyl acetate, and $(\square)$ benzyl benzoate at $T=298.2 \mathrm{~K}$. 


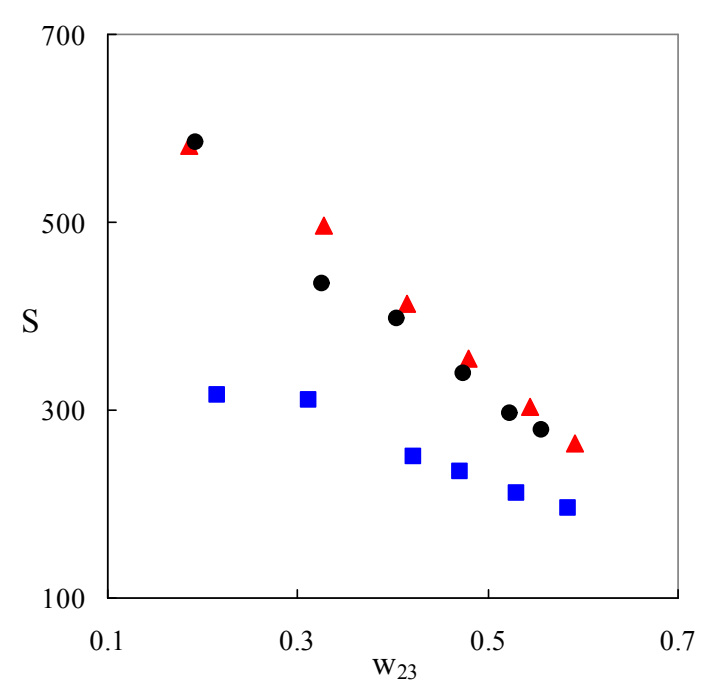

(a)

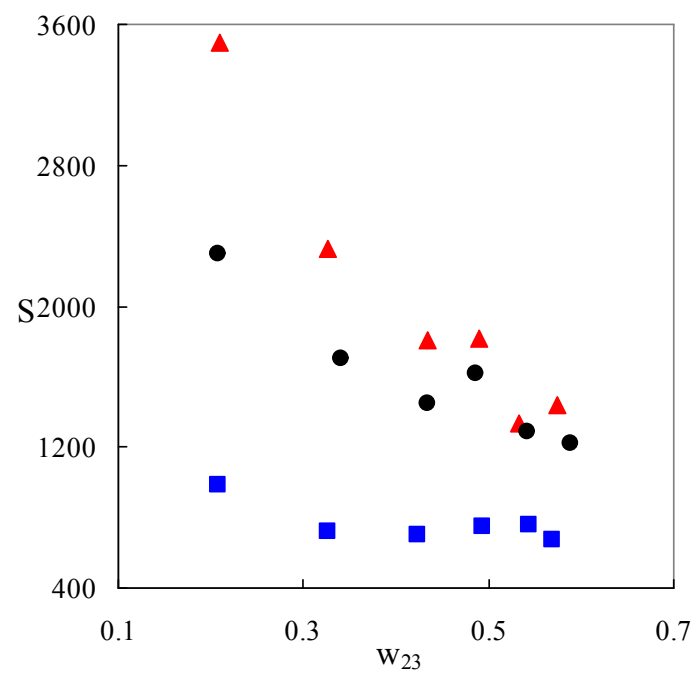

(b)

Figure S2 Variation of the separation factor $(\mathrm{S})$ of the acids with $w_{23}$ for the fwater $-(\mathbf{a})$ valeric acid or $(\mathbf{b})$ caproic acid - non-aliphatic ester) ternary systems; $(\bullet)$ cyclohexyl acetate, $(\boldsymbol{\square})$ benzyl acetate, and $(\boldsymbol{\Delta})$ benzyl benzoate $T=298.2 \mathrm{~K} p=101.3 \mathrm{kPa}$. 